(Aus dem Krüppelheim Stifung Dormagen, Köln-Merheim. [Leitender Arzt:

Dr. H. Landwehr.])

\title{
Zwei Beiträge zur Ätiologie der Osteochondritis juvenilis.
}

Von

\author{
Dr. med. H. Landwehr.
}

Mit 2 Abbildungen im Toxt.

(Eingegangen am 24. Juli 792:.)

Nach Dispositionsalter, Sitz, Vorgeschichte und Heilverlauf bieten die Krankheitsbilder der Spätrachitis im allgemeinen, der Schlatterschen, Köhlerschen und Perthesschen Krankheit soviel Wesensverwandtes, dals sie alle alsbald nach Bekanntwerden zu einem Bilde zusammengereimt und diese Versuche der Ärztewelt auch weitgehend mitgeteilt wurden. Wünschenswert ist die Kenntnis vor allem aus dem praktischen Grunde, damit Verwechslungen mit schwerwiegenclen Leiden tunlichst vermieden werden. Für die Wissenschaft darf aus der Beschäftigung besonders cine allmähliche Klärung der Frage nach den erregenden Trsachen erhofft werden. In dieser Hinsicht sind weiter auseinandergehende Heinungen kaum möglich; Vererbung, Enuchoidismus, Trauma, Tuberkulose sind Erklärungen, wie sie grundverschieclener kaum scin können.

Zwei meiner Beobachtungen lassen vor allem trophische und rein mechanische Ursachen gelten; sie beleuchten die Unklarheiten außergewöhnlich scharf, weshalb ich sie bekannt gebe.

1. Kind Ja., männlich, I Jahr alt; in der Geburt Verletzung beider Oberschenkel. Befund: Linker Arm leieht einwärts rotiert gehalten; Beweglichkeit in ubrigen gut. Seblaffe Lähmunư des linken Quadrize ps, GesiäBnuskulatur beiclerseits schlecht entwickelt. Peechtes Bein liegt völlig nuch außen rotiert. Ö́dem beiđer Füße bis iuber die Knö̈chel. Röntgenbild zeigt leicht winkelige Knickung des Oberschenkels $2^{1 / 2} \mathrm{~cm}$ unterbalb des kleinen Rollhügels. Der Schatten eines Kopfkerns ist auf dieser Seite nicht vorhanden. während er auf der anderen in Ërbsengröße deutlich und scharf unrandet sichtbar ist. Verdacht auf Schädigung in beiden Darmbein-Kreuzbeingelenkeu (s. Röntgenbild Abb. 1).

2. Find Lä., weiblich, 12 Jahre alt; in Anstaltsbehandlung wegen Kinderlähmung seit Sept. 1919; von anderer Seite ist früber u. a. eine Arthrodese rles linken Kuiegelenks mit Drahtnaht vorgenommen worden. Bei Aufnahme geringe Kontrakturstellung des Gelenkes. Diese hat im Laufe der Zeit (das Kind geht mit FuBunterschenkelschiene umber) eine Vermehrung erfahren. Seit Frühjahr 1921 klagt es zunehmend über Beschwerden beim Gehen in der Vorderseite des Knies; nur bei Bettruhe schmerzfrei. For Röntgenbild vom April 1921 war der Verdacht eines Fungus nicht ron der Hand zu weisen; es bestand wechseind starke Weichteilschwellung besonders an der Außenunterkante der Kniescheibe. Druck- und spontane Schmerzhaftigkeit an der Tuberositas tibiae. Rüntgenbild s, Abb. 2: Distale Femurepiphysenlinie auf der Streckseite auffallend stark klaffend; hier unscharfe 
Fontur der Diaphyse, sieht wie angenagt aus, während die Epiphyse in Seitenanfnalume sie zungenförmig überlagert. An Stelle der Tub. tibiar finclet sich ein im Verhältnis zum

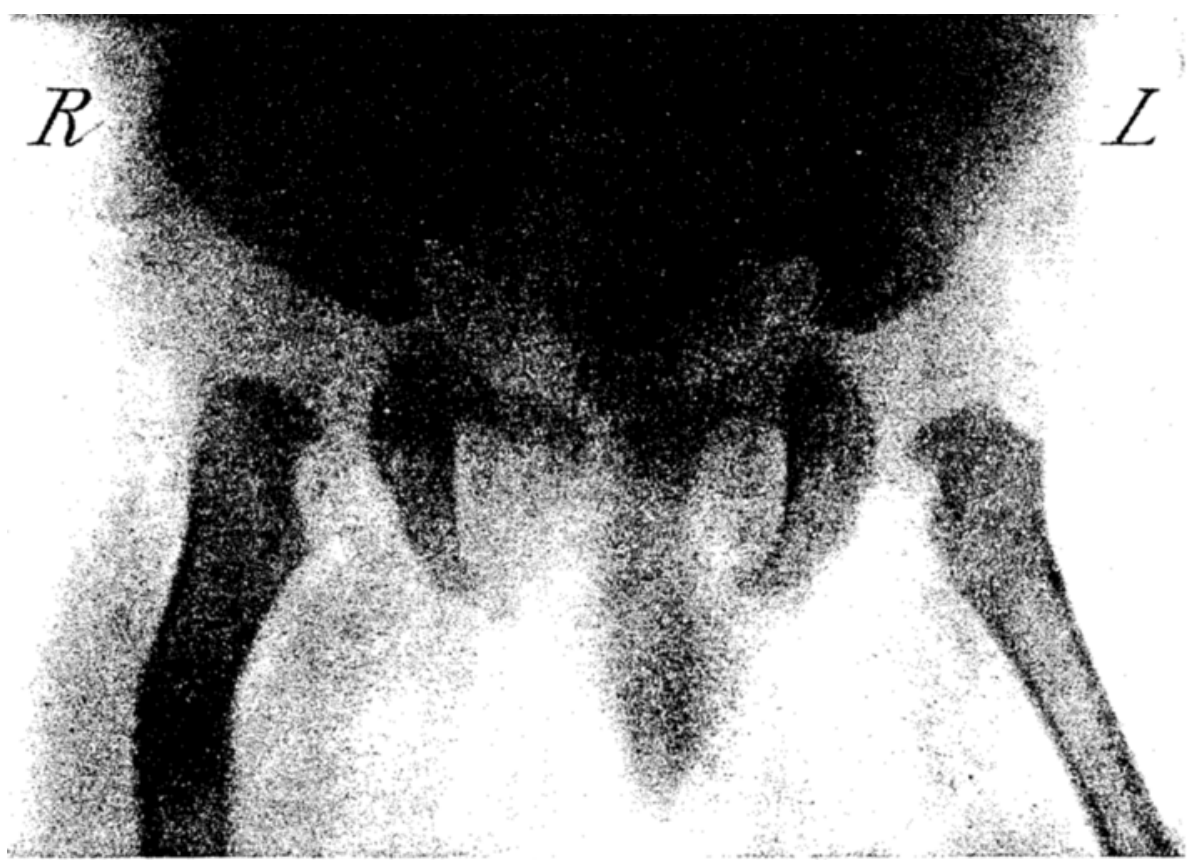

AbB.. .

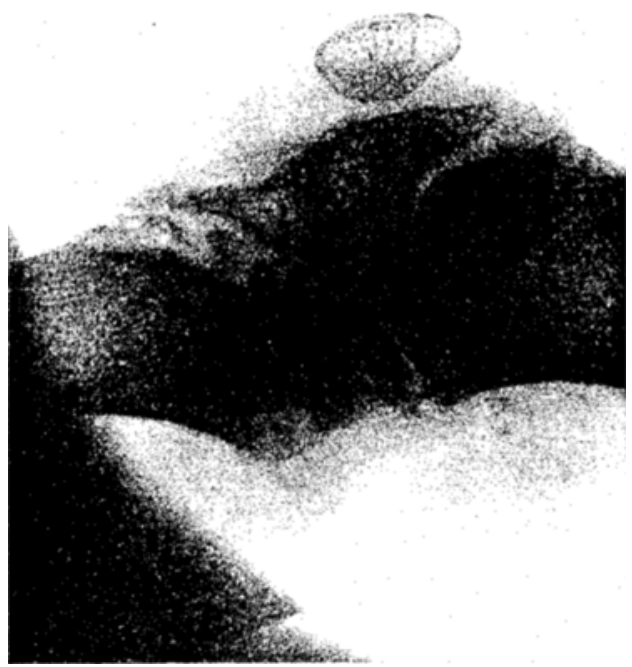

Abb. $\stackrel{2}{-1}$

Schaft stark aufyelelltex selutiten, ron wolkigem Bau. Einzclmassen zum Teil scharf abgerrenzt. Dex sichienbeinsibuat ist wie dor oberschenkelichaft angenart, die aufgehellte zone erstreckt sich fast bis zur Witte des Forderrückclurehmussors der Epiphysenscheiler. Bis Ende 1921 wurdem Gipsrerbande mit Gehbiugel getragrn. Obshon diesp eine Entlastung der Quadrizepsedune nieht abwichtich gaben, war mit ihnen dere Gang dech völly schmerzlos; nach jecler $A b$ nahme starke schmerzen schoon nach den ersten siduritten. Nach subjebtiver Heilungr wurcle ein Röntgenbild ange. fertigt. clas völlige Iückkehr zur Norn an der fraglichen Stelle zeigt; während der ganzen Krankheitsdauer bot das llädchen im übrigen beinerlei auffallige. allgemeine Krankheitszeicben; gelegentliche Spuren von Temperatur. crhöhung komiten mit den örtlichen Leiden nicht in Zusammenhang gebracht werden.

Die Cmstände, unter denen der 2. Fall zur Bcobachtung gekommen ist, können dazu veranlassen, die Krankhcitsursache in einer ganz bestimmten 
Richtung zu suchen. Es handelt sich um ein seit Jahren in Anstaltspflege stehendes Kind, bei dem sich das Knieleiden erst während dieser Zeit entwickelt. Auf diese Tatsache glaube ich mich beim Ausschluß von "Spätrachitis" stützen zu können. Als disponicrender Zustand kömte diese vielleicht in Anspruch genommen werden, so, wie dies auch bisher von manchen Beobachtern mit vollem Recht geschehen ist; der ursïchliche $\mathrm{H}$ a u pt faktor würde dann , abnorme statische Verhältnisse" heißen.

Abgesehen von den, unten ausführlicher zu erwähnenden, ganz vereinzelt beobachteten Affektionen des oberen Humerusendes, spielen bei der Osteochondritis stets mechanische Momente ohne Zweifel eine bedeutsame Rolle; manchen Beobachtern haben sie allein in ursächlicher Beziehung genügt. Um wieviel mohr darf man sich in vorliegendem Falle damit zufrieden geben. Weil die statisch-mechanischen Verhältnisse hier primär so sehr gestört waren, fielen vielleicht auch die klinischen Zeichen, einschließlich Röntgenhild, so deutlich aus. Und doch wollen die klaren und glaubwürdigen Beschwerden in den Rahmen statisch bedingter Prozesse nicht hineinpassen. Die unmittelbar nach Abnahme von Dauerverbänden wieder auftetende Schmerzhaftigkeit erhcischt rein klinisch eine (Xrundlage entzündlicher Art. Auch Kreutzer will diese' (d. h. Infektion) in der Pathogenese der Osteochondritis nicht außer acht lassen. wiewohl er dem Trauma hohe Bedeutung zumißt.

Der an erster Stelle beschriebene Fall wirft ein Schlaglicht auf clie bei angeborener Hïftgelenkverrenkung nach der Repusition und bei Gelenkverletzungen beobachteten und mit der Osteochondritis in Zusammenhang gebrachten Veränderungen. Jie Ausbeute an klarem, derartigen Material ist hisher gering. Boi obiger Beobachtung l bietet die Kopfepiphyse gewisses Interesse. Der in der Regel in lo. Lebensmonat erscheinende, und hier auf der gesunden Sxite sichtbare Kern des Schenkelkopfes ist an dem intra partum gebrochenen Oberschenkel nicht sichtbar. Es scheint mir nicht schwer, den Zusammenhang diescr Erscheinungen zu erklären, wobei mir Feststellungen gclegentlich der vor Jahren lebhaften Aussprache über das novellenhaft klingende Thema "frakturloser Kallus" erinnerlich werden: Die Myositis ossificans tritt fast nur bei kräftigen, meist männlichen Individuen in Erscheinung. Beim wachsenden Organismus ist sie unbekannt; es zeigt sich in dieser Abhängigkeit eine feste Tendenz der Knochenbildung, eine Affinitat im Mineralstoffwechsel. Daßs bei Fall 1 die Knochenbildungstendenz in der Richtung auf den (übrigens fast nicht behandelten) Oberschenkelbruch stärker war, als zu der, vielleicht auch irgendwie unmittelbar geschädigten, gleichseitigen Kopfepiphyse hin, ist durchaus vorstellbar. Leider kann ich die Frage, wann und wie die Weiterentwicklung des Kopfkernes vonstatten ging, nicht beantworten, weil eine Nachuntersuchung bisher nicht zustande kam. Immerhin ist der große Rückstand in der Entwicklung unter dem Gesichtswinkel der Osteochondritis von Interesse. Eine gewisse Parallele bietet die Veröffentlichung von Hackenbroch, wenn ich auch der Ansicht bin, daß der Kausalzusammenhang OperationstraumaKopfkernschaden nicht über jeden Zweifel erhaben ist, vielleicht sogar insofern in sein Gegenteil verkehrt werden kann, als die Beschwerden der bereits beginnenden Osteochondritis den Kranken in die Behandlung geführt haben. 
Wie mir die burchsicht des Schriftums zeigt, ist es nicht das crste hal, (lak hier Gel)urtsverletzungen im Gelenkbereich in Beziehung gebracht werden zur Osteochondritis juvenilis. In überzeugender Weise hat Valeutin in der Gruppe der früherworbenen Schulterdeformitäten mit der billigen Erklärung durch Luxation oler Epiphysenlösung aufgeräumt. Bei cliesem, keine Stütztätigkeit ausübenden Skeletteil scheinen ihm Beziehungen zu bestehen zur Osteochondritis. Andere und seine eigenen Fälle zeigen später im Röntgenbild "Knochenkern der Epiphyse viel kleiner als auf der gesunden Seite" oler, ,starker Grïßenunterschied zwischen beiden Humerusepiphysen zu ungunsten der verlatzten Seite" oxler ",starke Abflachung und Verschmälerung des Kopfteiles" ofler , mit zunehmendem Alter bleiben die Epiphysenkerne im Größenwachstum zurück und zeigen unregelmäßige Unrisse": oder :, Epiphyse in toto verkleinert, atrophisch und etwas deformiert". Falentin beantwortet die Frage nach der letzten anatomischen Ursache dieser Kopfkernveränderungen so, wie sich für die Schulterdeformitäten geradezu von selbst ergibt; es stehen doch bei diesem Krankheitsbild die Plexusschärligungen völlig im Vordergrum. Dic Kapsel hat aus diesem ihre Versorgung hauptsächlich durch Nervus axillaris und suprascapularis (Auerbach).

Im allgemeinen kann außer dieser neurotischen Atrophie, ferner der von mir oben supponierten, als reaktiv zu bezeichnenden Entwicklungsstörung, drittens rlie trophische in den Füllen kaum vernachlässigt werden, in welchen sog. Epiphysenlösung wirklich bestand oder sonstwie ernährende Gefäbe Schaden litten. Nach Perthes' Ansicht sind diese Störungen der zum Schenkelkopf tretenden Arterie eine regelmäßige pathologisch-anatomische Beobachtung bei der Osteochondritis.

Im Hinblick auf die bestehenden motorischen Ausfälle im obigen Falle 1 ist die Kritik wohl berechtigt, für die Vcrzögerung der Kopfkernentwicklung neurotische Einflüsse anzuschuldigen. Der Röntgenschatten des zugehörigen Schaftes und des Halses gibt beim Vergleich mit der gesunden Seite in dieser Richtung keine weiteren Anhaltspunkte. Als lädierte Nerven kommen der Nervus obturatorius und ein akzessorischer Ast desselben in Betracht.

Bis zur Keruntnis der beiden oben veröffentlichten Fälle war ich durchaus geneigt, mich der Meinung derer anzuschließen, die die Osteochondritis und ihre Verwandten in erster Linic als Außerungen des rachitischen Kranhhcitsprozesses betrachten. Heute int mir das nicht mehr möglich, da im Falle 2 nächst statisch-mechanischen Ursachen symptome entzündlichen Charakters, im Falle I primär-trophische Einflüsse ausschlaggebende Wertung in der Ätiologiefrage verlangen. Auf Grund der bisherigen klinischen Beobachtung (Valentın, Haß, obige Fälle) müıssen Ernährungsanomalien neurotrophischer Art weit in den Vordergrund gestellt verden.

\section{Literatur.}

1. Hackenbroch, Zur Itiologie der Osteoarthritis deforman: juvenilis des Hüf. gelenks. Zentralbl. f. Chirurg. 1921. - 2. Erkes, Chirurg. Kongr. 1921. -.. 3. Valentin, Münch. med. Wochenschr. 1921. 820. - 4. Derselbe, Arch. f. orthop. Unfallchirurg. 19. - 5. Kreutzer. Zur Ätiologie und Pathogeneso der Osteochondritis deformans juvenilis coxae. Zentralbl. f. Chirurg. 1920. - 6. Haß, Über die sogenarnte Osteochondritis deformans. Zentralbl. f. Chirurg. 30. 1921. 1089. 